\title{
Yin Yang 1 (YY1) synergizes with Smad7 to inhibit TGF- $\beta$ signaling in the nucleus
}

\author{
YAN XiaoHua $^{\dagger *}$, PAN Jun ${ }^{\dagger}$, XIONG WanWan, CHENG MinZhang, SUN YingYuan, \\ ZHANG SuPing \& CHEN YeGuang
}

State Key Laboratory of Biomembrane and Membrane Biotechnology, Tsinghua-Peking Center for Life Sciences, School of Life Sciences, Tsinghua University, Beijing 100084, China

Received October 16, 2013; accepted November 19, 2013; published online December 23, 2013

\begin{abstract}
As a prototype of the TGF- $\beta$ superfamily cytokines, TGF- $\beta$ is well known for its diverse roles in embryogenesis and adult tissue homeostasis. TGF- $\beta$ evokes cellular responses by signaling mainly through cell membrane receptors and transcription factor R-Smads and Co-Smad (Smad4), while an inhibitory Smad, Smad7, acts as a critical negative regulator of TGF- $\beta$ signaling. Smad7 antagonizes TGF- $\beta$ signaling by regulating the stability or activity of the receptors or blocking the DNA binding of the functional R-Smad-Smad4 complex in the nucleus. However, the function of Smad7 in the nucleus is not fully understood. Yin Yang 1 (YY1) is a ubiquitously expressed transcription factor with multiple functions. It has been reported that YY1 can inhibit Smad-dependent transcriptional responses and TGF- $\beta$ /BMP-induced cell differentiation independently of its DNA binding ability. In this study, we found that Smad7 interacts with YY1 and the interaction is attenuated by TGF- $\beta$ signaling. Reporter assays and target gene expression analyses revealed that Smad7 and YY1 act in concert to inhibit TGF- $\beta$-induced transcription in the nucleus. Furthermore, Smad7 could enhance the interaction of YY1 with the histone deacetylase HDAC1. Consistently, YY1 and HDAC1 augmented the transcription repression activity of Smad7 in Gal4-luciferase reporter analysis. Therefore, our findings define a novel mechanism of Smad7 and YY1 to antagonize TGF- $\beta$ signaling.
\end{abstract}

TGF- $\beta$, Smad7, YY1, HDAC1, signaling regulation, transcriptional co-repressor

Citation: $\quad$ Yan XH, Pan J, Xiong WW, Cheng MZ, Sun YY, Zhang SP, Chen YG. Yin Yang 1 (YY1) synergizes with Smad7 to inhibit TGF- $\beta$ signaling in the nucleus. Sci China Life Sci, 2014, 57: 128-136, doi: 10.1007/s11427-013-4581-2

TGF- $\beta$ (transforming growth factor-beta) is a prototype of the large TGF- $\beta$ cytokine superfamily, which comprises 33 members in mammalian cells, including TGF- $\beta$, activin, nodal, BMPs (bone morphogenetic proteins), GDFs (growth differentiation factors) and others [1-4]. Since its first discovery in the early 1980 s, TGF- $\beta$ has attracted much attention due to its diverse roles in regulating cellular activities, such as cell proliferation and differentiation, apoptosis and senescense, cell matrix remodeling, adhesion and migration [5-7]. It is well established that TGF- $\beta$ is a critical player in

$†$ Contributed equally to the work

*Corresponding author (email: yanxh@ mail.tsinghua.edu.cn) embryogenesis and adult tissue homeostasis. To achieve this, TGF- $\beta$ signal transduction is finely controlled tempospatially via multiple mechanisms, and dysregulation of TGF- $\beta$ signaling is closely associated with a wide array of human disorders including tumorigenesis, tissue fibrogenesis and developmental defects [2,8-10].

TGF- $\beta$ signaling is initiated when the ligands bind to their cognate cell membrane serine/threonine kinase receptors, the type II TGF- $\beta$ receptor T $\beta$ RII and the type I receptor T $\beta R I$, where T $\beta R I$ is phosphorylated and activated by T $\beta$ RII $[4,11]$. Then signaling is propagated to downstream Smad proteins, although some other signaling molecules (MAPKs, PI3K/Akt, PAK2, RhoA, LIMK1/2, etc.) are also 
reported to be activated by TGF- $\beta$ in different circumstances or cell types [12]. The Smad protein family members are divided into three groups based on their structures and functions. Upon TGF- $\beta$ stimulation, R-Smads (receptorregulated Smads, Smad2/3 for TGF- $\beta$ ) are phosphorylated directly by T $\beta R I$ at the carboxyl terminal SXS motif, oligomerize with Co-Smad (common Smad, Smad4) and accumulate together in the nucleus, where they bind DNA and regulate target gene expression in collaboration with other transcription factors or co-factors $[11,13]$.

Smad6 and Smad7 constitute the third group of the Smad family, I-Smads (inhibitory Smads), functioning as critical negative regulators of the TGF- $\beta$ superfamily signaling $[14,15]$. They share conserved C-terminal MH2 domains with R-Smads and Smad4, but the N-terminal domains are more divergent. Gene targeting and transgenic studies in mouse models clearly affirmed the significant roles of Smad7 in embryonic development, tissue maintenance and disease progression [15-18]. Biochemical studies revealed that multiple mechanisms can be exploited by Smad7 to inhibit TGF- $\beta$ signaling. In the cytoplasm, Smad7 forms a complex with TGF- $\beta$ receptors and recruits E3 ubiquitin ligases, phosphatases and other regulators, thus affecting receptor stability or activity $[2,14,19]$. We have reported that in the nucleus, Smad7 can bind DNA in a sequencespecific manner and inhibit TGF- $\beta$ signaling by impeding functional Smad-DNA complex formation [20,21]. Smad7 also interacts with histone deacetylases (HDACs), E2F1 and SIRT1, implying that Smad7 might act as a transcriptional repressor/co-repressor and regulate gene expression epigenetically [22-24]. Interestingly, Smad7 can also associate with the transcription factor IRF1 or MyoD, and behave as a co-activator [25,26]. Similarly, Smad6 acts as a co-repressor by associating with HDACs, Hoxc- 8 or CtBP in the BMP pathways [27-29]. In addition to Smad6/7, HDACs can also interact with and be recruited by other Smad proteins, mediating Smad-induced transcriptional repression [13].

Yin Yang 1 (YY1) is a multifaceted nuclear protein first cloned and characterized in 1991 [30-32]. Early studies indicated that YY1 is a ubiquitously expressed and evolutionarily conserved transcription factor, containing four $\mathrm{C} 2 \mathrm{H} 2$-type zinc fingers at the $\mathrm{C}$-terminus and histidine/acidic amino acid-rich regions at the $\mathrm{N}$-terminus. The zinc fingers mediate specific DNA binding, while both of the $\mathrm{N}$ - and C-terminal domains are required for its transcriptional activities. As the name implies, YY1 can either activate or repress gene expression, depending on associated proteins and/or cell types. Several histone modifiers have been documented to associate with YY1. Recruitment of co-activators p300, CBP, PCAF or PRMT1 could lead to target gene activation via histone modifications, while recruitment of co-repressors HDACs, Ezh1/2 or DNMTs would result in histone deacetylation, H3K27 methylation or promoter hypermethylation, and finally gene suppression.
In addition, several recent studies showed that YY1 may affect gene expression independently of its DNA binding ability. YY1 associates with androgen receptor (AR) and stimulates the expression of prostate-specific antigen (PSA) in prostate cancer cells, and a YY1 mutant deficient in DNA binding is similarly effective [33]; in addition, YY1 acts as a co-repressor for the transcription factor HOXA11, GON4L and ATF/CREB [34-36]. Likewise, YY1 interacts with R-Smads (Smad1/2/3) and Smad4, inhibiting Smadmediated transcriptional responses by disrupting SmadDNA binding, thus interfering with TGF- $\beta / B M P-$ stimulated cell differentiation; all of these effects do not depend on the DNA binding activity of YY1 [37]. YY1 was also shown to work with Smad1/4 in BMP-induced heartspecific expression of CAR3 in chicks [38]. However, the interplays between YY1 and the TGF- $\beta$ /BMP pathways are not fully understood.

Although the nuclear functions of Smad7 have been exemplified by several studies, the underlying mechanisms and regulatory mechanisms remain elusive. In this study, we characterized the interaction between Smad7 (also Smad6) and YY1, which could be attenuated by TGF- $\beta$ signaling. Reporter assay and in vivo target gene expression analyses revealed that YY1 and Smad7 cooperate with one another to inhibit TGF- $\beta /$ Smad-driven transcription, while knockdown of endogenous YY1 or Smad7 attenuates the inhibitory effects of the other protein. Mechanistically, Smad7 is capable of promoting the YY1-HDAC1 association in the nucleus, consistent with that YY1 and HDAC1 facilitate the transcription repression activity of Smad7 in Gal4-luciferase reporter assay. Together, these results define a novel mechanism for TGF- $\beta$ signaling regulation.

\section{Materials and methods}

\subsection{Cell culture and transfection}

Human embryonic kidney (HEK) 293T cells, 293FT cells and Hep3B hepatocellular carcinoma cells were grown in DMEM (Dulbecco's Modified Eagle Media) supplemented with $10 \%$ fetal bovine serum (FBS) at $37^{\circ} \mathrm{C}$ in a humidified, $5 \% \mathrm{CO}_{2}$ incubator. Transfection was accomplished with VigoFect (Vigorous Biotechnology, Beijing, China) or Lipofectamine 2000 (Invitrogen, USA) according to the manufacturer's instructions.

\subsection{Plasmids and antibodies}

YY1 constructs were generously provided by Dr. Shi Yang (Harvard Medical School, USA) and Gal4-TK-luciferase reporter was a gift from Dr. Feng XinHua (Baylor College of Medicine, USA). The HDAC1 coding sequence was cloned into pcDNA3; Gal4-DBD (DNA binding domain)-fused Smad7 construct was generated based on pcDNA $3.1^{+}$, and other protein-coding constructs and Smad7 
shRNA plasmid were described previously [20,39,40]. siRNAs targeting human YY1 were designed as GGGAGCAGAAGCAGGUGCAGAUdTdT, GCAAGAAGAGUUACCUCAGdTdT and GGCAGAAUUUGCUAGAAUGdTdT for siRNA 1 to 3, respectively. Smad7 antibody was generated by immunizing rabbits with the N-terminal domain of Smad7 (amino acids (aa) 1-259). Anti-Flag antibody (M2) was purchased from Sigma (St. Louis, USA), and antibodies against YY1 and other tags were from Santa Cruz Biotechnology (California, USA).

\subsection{Reporter assay, immunoprecipitation and im- munoblotting}

Reporter assay, immunoprecipitation and immunoblotting were performed as previously described [39]. Reporter assays were performed in triplicate, and the data are presented as mean \pm standard deviations (SD) after normalization to Renilla-luciferase activity.

\subsection{RNA isolation and quantitative RT-PCR}

Total RNA extraction from cultured monolayer cells and quantitative reverse transcription-PCR (RT-PCR) were performed as previously reported [40,41]. The primers used were as follows: for human GAPDH (glyceraldehyde-3phosphate dehydrogenase), 5'-ACCACAGTCCATGCCATCAC-3' and 5'-TCCACCACCCTGTTGCTGTA- 3'; for PAI-1, 5'-GAGACAGGCAGCTCGGATTC-3' and 5'-GGCCTCCCAAAGTGCATTAC-3'; for FURIN, 5'-CCTGGTTGCTATGGGTGGTAG-3' and 5'-AAGTGGTAATAGTCCCCGAAGA-3'; for INHBE, 5'-ATCTTCCGATGGGGACCAAG-3' and 5'-AGAGTTAAGGTATGCCAGCCC-3'; for SnoN, 5'-AGAGACTCTGTTTGCCCCAAGT-3' and 5'-CATGCTAAACTTCTCCTTCATTTC-3'; and for $F S T, \quad 5^{\prime}$-ACGTGTGAGAACGTGGACTG-3' and 5'-CACATTCATTGCGGTAGGTTTTC-3'. Gene expression was normalized against GAPDH mRNA. Each sample was measured in triplicate experiments. Data were analyzed using Microsoft Excel software.

\subsection{Lentivirus production and infection}

The coding sequences (CDS) of GFP, Smad7 and YY1 were cloned into pENTR1A plasmid and LR clonase reactions (Invitrogen, USA) were carried out to place these CDS under the control of CMV promoter in the p2k7neo lentiviral backbone (a gift from Dr. Kehkooi Kee, Tsinghua University) [42]. To produce defective lentivirus, we transfected 293FT cells using Lipofectamine 2000 with the above recombinant lentivirus construct, pCMV $\Delta 8.9$ and pMD.G (VSVG), at a ratio of $1: 1.5: 1$. The culture supernatants were collected at 3 days post-transfection, and viral particles were concentrated by centrifugation. Hep3B cells were infected with lentivirus particles in the presence of polybrene (final concentration of $9 \mu \mathrm{g} \mathrm{mL}^{-1}$, Sigma). Two or three days later, infection efficiency was examined on the basis of GFP expression and immunoblotting. For GFP- and Smad7-stable cell line establishment, G418 $(250 \mu \mathrm{g} \mathrm{mL}$ for final concentration; Invitrogen, California, USA) was added into culture media for $5 \mathrm{~d}$ to select cells with stable viral integration.

\section{Results}

\subsection{YY1 is a novel binding partner of Smad7}

Smad7 plays a crucial role in balancing TGF- $\beta$ signaling via negative feedback loops [14]. In order to explore how Smad7 activity is controlled, we searched for its interacting proteins. GST (glutathione S-transferase)-tagged Smad7 was overexpressed in HEK293T cells, and after GST pull-down, all the Smad7-associated proteins were subjected to SDS-PAGE gel analysis followed by mass spectrometric identification of Smad7-bound proteins. The nuclear transcription factor YY1 was identified. To verify their interaction, we introduced constructs encoding HA-tagged YY1 and various Myc-tagged Smads into 293FT cells. At $40 \mathrm{~h}$ post-transfection, the cells were lysed and subjected to anti-HA immunoprecipitation followed by anti-Myc immunoblotting. In accordance with previous report [37], YY1 immunoprecipitated all the tested R-Smads, Smad4, and also the two inhibitory Smads, Smad6/7 (Figure 1A). Interaction of YY1 with Smad6/7 seemed to be stronger than those with R-Smads or Smad4 after normalizing the total protein levels. Smad7 is also associated with YY1 at the endogenous level in 293FT (Figure 1B). Interestingly, their interaction was attenuated by TGF- $\beta 1$ treatment, implying that this interaction is regulated by TGF- $\beta$ signaling.

To consolidate the above phenomenon, we tested the interaction of ectopic YY1 and Smad7 in the presence or absence of constitutively active form of TGF- $\beta$ type I receptor ca-T $\beta$ RI/ALK5, which is able to activate R-Smads and elicit signal transduction [43]. The immunoprecipitation result showed that YY1-Smad7 interaction was apparently weaker when ca-T $\beta R I$ was co-expressed (Figure 1C), and similar result was obtained when a constitutively active BMP type I receptor ca-BMPRIB/ALK6 was co-transfected along with YY1, Smad6 or Smad7 (Figure 1D). The reverse immunoprecipitation assay gave rise to the consistent results (Figure 1E). These results clearly demonstrate that binding of YY1 to I-Smads is negatively regulated by TGF- $\beta$ or BMP signaling. The $\mathrm{N}$-terminal region and the conserved $\mathrm{C}$-terminal MH2 domain of Smad7 could exert different effects in protein interaction or signaling regulation, thus we continued to determine which domain is responsible for Smad7 interaction with YY1. As shown in Figure 1F, YY1 is mainly associated with the $\mathrm{MH} 2$ domain of Smad7. 

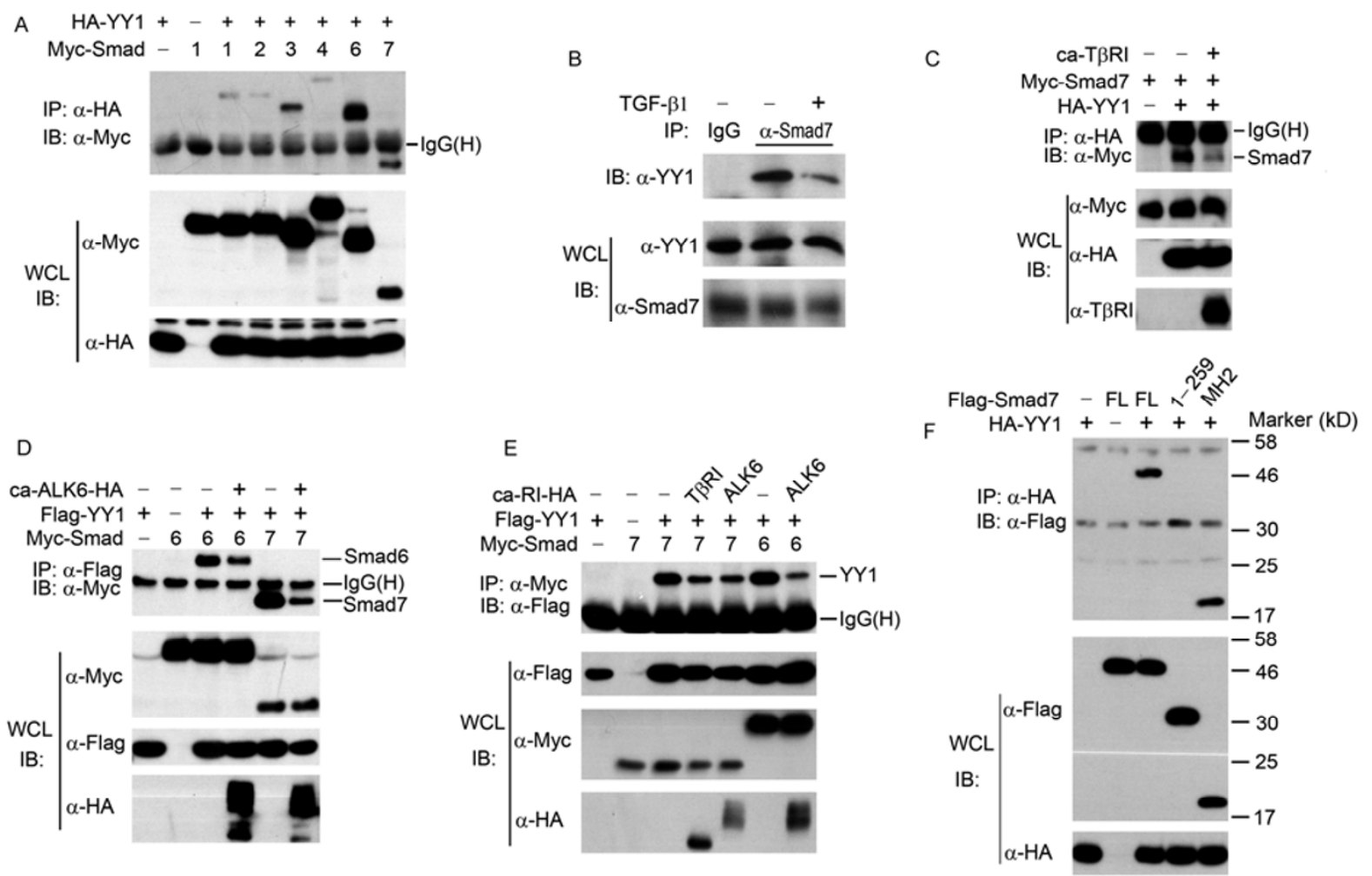

Figure 1 Smad7 interacts with YY1. A, YY1 associates with Smad proteins. HA-YY1 and Myc-Smad constructs were transfected into 293FT cells. At $40 \mathrm{~h}$ post-transfection, cells were lysed in TNE buffer and cell lysates were subjected to anti-HA immunoprecipitation (IP) followed by anti-Myc immunoblotting (IB), and protein expression in whole cell lysates (WCL) was examined by immunoblotting using antibodies against Myc- and HA-tags respectively. B, Endogenous interaction between Smad7 and YY1. Confluent 293FT cells were treated with or without $100 \mathrm{pmol} \mathrm{L}^{-1}$ TGF- $\beta 1$ for $2 \mathrm{~h}$. Then cells were lysed for IP using anti-Smad7 antibody or control rabbit IgG and anti-YY1 immunoblotting. C-E, TGF- $\beta$ and BMP signaling attenuates the Smad7-YY1 interaction. Various constructs were introduced into 293FT cells as indicated, and immunoprecipitation and immunoblotting were carried out as in Figure 1A. F, Domain mapping of Smad7. The experiment was done as in Figure 1A. 1-259, the N-terminal region of Smad7 spanning amino acids (aa) 1-259; MH2, the MH2 domain of Smad7 (260-426 aa).

\subsection{YY1 cooperates with Smad7 to inhibit TGF- $\beta$ - induced transcriptional responses}

Smad7 is well documented to antagonize TGF- $\beta$ signaling, and YY1 was also reported to inhibit TGF- $\beta$-induced transcription by associating with R-Smads and Smad4 [37]. The identification of the YY1-Smad7 association prompts us to ask whether the two proteins could cooperate with one another to negatively regulate TGF- $\beta$ signaling. To address this question, we first performed reporter assays in 293FT cells using the CAGA-luciferase reporter, which contains repeated canonical Smad binding elements (SBE) and responds to TGF- $\beta$ quite specifically [44]. As shown in Figure $2 \mathrm{~A}$, various amounts of Smad7- or YY1-expression constructs were transfected together with CAGA-luciferase reporter and the control Renilla plasmid. Both Smad7 and YY1 inhibited TGF- $\beta$-stimulated reporter expression in dose-dependent manners, and Smad7 seemed to be more effective in this respect. Then we transfected low amounts of Smad7 or YY1 constructs alone or together and carried out reporter assay experiment. Although Smad7 or YY1 alone decreased TGF- $\beta$-triggered transcriptional responses to a certain degree, co-expression of the two proteins led to a dramatic inhibition (Figure 2B), indicating that YY1 could cooperate with Smad7 to inhibit TGF- $\beta$-elicited transcription.

To confirm the cooperative effect between Smad7 and YY1 at the endogenous level, shRNA targeting Smad7 and siRNAs targeting human YY1 were used. Two of the three siRNAs (si-1 and si-3) designed showed high efficiency in knocking down YY1 expression (Figure 2C). Knockdown of YY1 alleviated the inhibitory effects of Smad7 to some extent, while silence of $\operatorname{Smad} 7$ attenuated the inhibitory effects of YY1 on TGF- $\beta$ signaling (Figure 2D).

\subsection{YY1 synergizes with nuclear Smad7 and facilitates its transcription repression activity}

Both YY1 and Smad7 are reported as primarily nuclear proteins [20,31], and this was confirmed in Hep3B hepatocellular carcinoma cells by immunofluorescence (data not shown). We reasoned that they may physically associate and functionally synergize in the nucleus. To examine this, we compared the synergy between YY1 and wild-type Smad7 or nuclear localization signal (NLS)-tagged Smad7, which is completely localized in the nucleus when ex- 

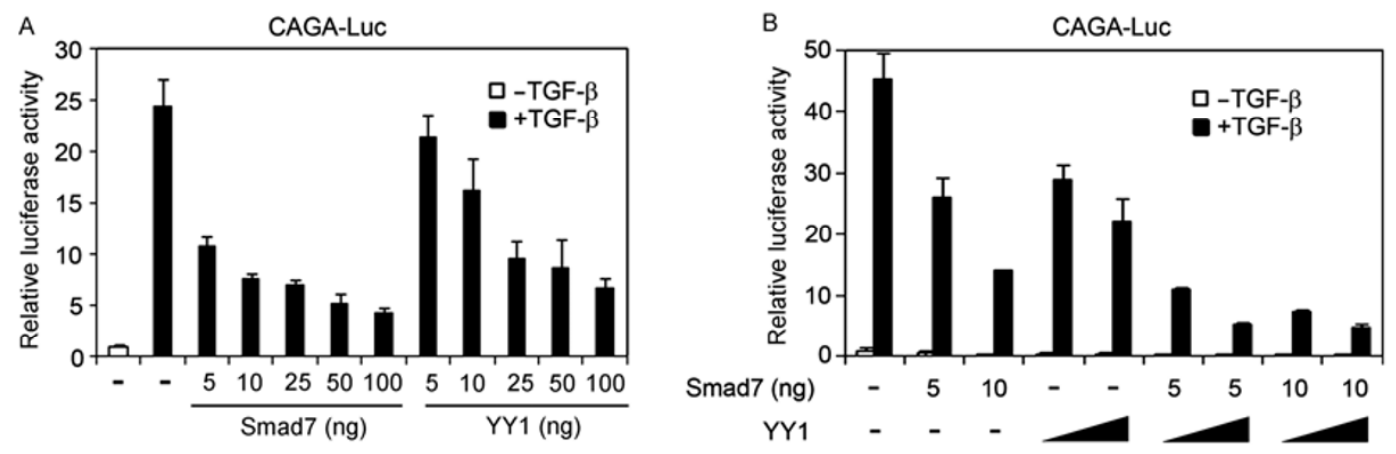

C

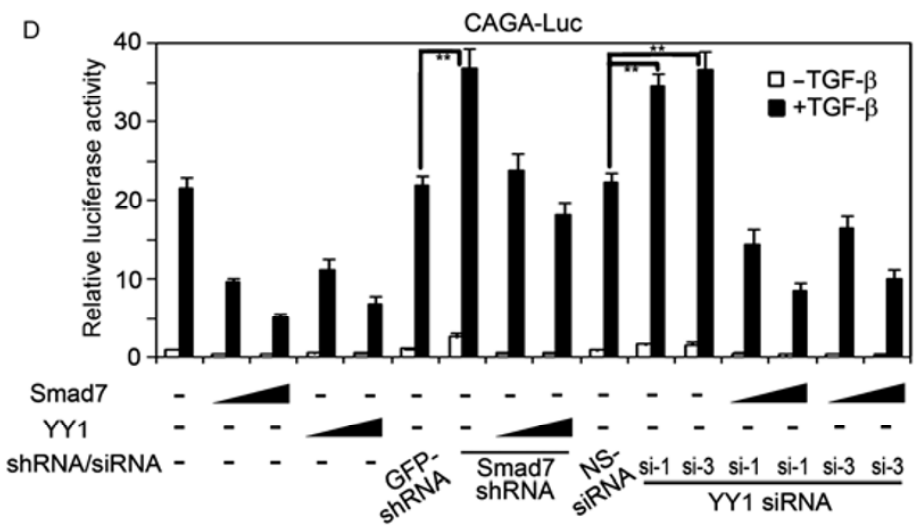

Figure 2 Smad7 and YY1 cooperate with one another to inhibit TGF- $\beta /$ Smad-driven transcriptional responses. A, Smad7 and YY1 antagonize TGF- $\beta$ /Smad-driven CAGA-luciferase reporter expression in a dose-dependent manner. One day after plating of 293FT cells into 24 -well plates, different amounts of Smad7 and YY1 constructs were transfected along with the CAGA-luciferase reporter (200 ng per 3 wells, the same for below) and Renillaluciferase plasmid $(20 \mathrm{ng})$. After $20 \mathrm{~h}$, the cells were treated with $100 \mathrm{pmol} \mathrm{L}{ }^{-1}$ TGF- $\beta 1$ overnight and harvested for luciferase activity measurement as described in Materials and methods. B, Smad7 cooperates with YY1 to inhibit TGF- $\beta$ signaling. Plasmids encoding Smad7 (5 and 10 ng), YY1 (10 and 25 ng), CAGA-luciferase (200 ng) and Renilla-luciferase (20 ng) were transfected into 293FT cells as indicated. C, GFP-expression plasmid, non-specific (NS-) siRNA or YY1-targeting siRNAs were transfected into 293FT cells using Lipofactamine 2000. Endogenous YY1 expression level was detected by immunoblotting. Tubulin was detected as loading controls. D, Both Smad7 and YY1 are required for efficient repression of TGF- $\beta$ signaling. Plasmids encoding YY1 (25 and $50 \mathrm{ng}$ ), Smad7 (25 and $50 \mathrm{ng}$ ), Smad7 shRNA (100 ng) or GFP-targeting shRNA (100 ng), NS-siRNA (100 ng) or YY1-specific siRNAs $(100 \mathrm{ng})$ were transfected into $293 \mathrm{FT}$ cells for CAGA-luciferase reporter assay. $* *, P<0.01$.

pressed in cells [20]. Indeed, CAGA-luciferase reporter assay in Hep3B cells revealed that NLS-Smad7 also worked together with YY1 in antagonizing TGF- $\beta$-induced transcriptional responses, as effective as that of wild-type Smad7 (Figure 3A), strongly suggesting that it is nuclear Smad7 that collaborates with YY1.

As a transcription factor/co-factor, YY1 and Smad7 can associate with histone deacetylases (HDACs) and regulate gene expression [22,24,31,32]. Therefore, we asked whether HDACs are involved in the synergy between YY1 and Smad7 in attenuating TGF- $\beta$ signaling. As a typical member, HDAC1 was reported to associate with both $\mathrm{Smad} 7$ and YY1 [22,31] (also our unpublished data), thus was tested here. First, we examined whether Smad7 could affect the association of YY1 with HDAC1. As shown in Figure 3B, YY1 immunoprecipitated both HDAC1 and Smad7, and co-expression of Smad7 strengthened the YY1-HDAC1 interaction. As Smad7 has been shown to possess transcription suppression ability using Gal4-luciferase reporter assay [45] (also our unpublished data), we determined whether YY1 or HDAC1 could facilitate Smad7. Expression of Gal4-DBD-fused Smad7 alone decreased the Gal4-TK- luciferase reporter expression to a certain degree, while co-expression of YY1 and/or HDAC1 further reinforced Smad7's effect (Figure 3C), implying that YY1 and HDAC1 could facilitate Smad7's function as a transcriptional co-repressor. Functionally, HDAC1 was able to attenuate TGF- $\beta$-induced CAGA-luciferase reporter expression moderately when expressed alone, and its co-expression with Smad7 and YY1 led to a dramatic inhibition of TGF- $\beta$ signaling (Figure 3D).

\subsection{Smad7 inhibits TGF- $\beta$-induced gene expression in Hep3B cells in cooperation with YY1}

Having established that YY1 cooperates with nuclear Smad7 to inhibit TGF- $\beta$-stimulated transcriptional responses, we wondered whether this cooperation is significant in vivo by examining TGF- $\beta$ target gene expression in Hep3B cells. Hep3B cells with stable expression of GFP or Smad7 were infected with or without YY1-expression virus. Three days later, the cells were harvested to detect $\operatorname{Smad} 7$ and YY1 expression by immunoblotting (Figure 4A). Then parallel samples were treated with or without TGF- $\beta 1$ for $4 \mathrm{~h}$ 


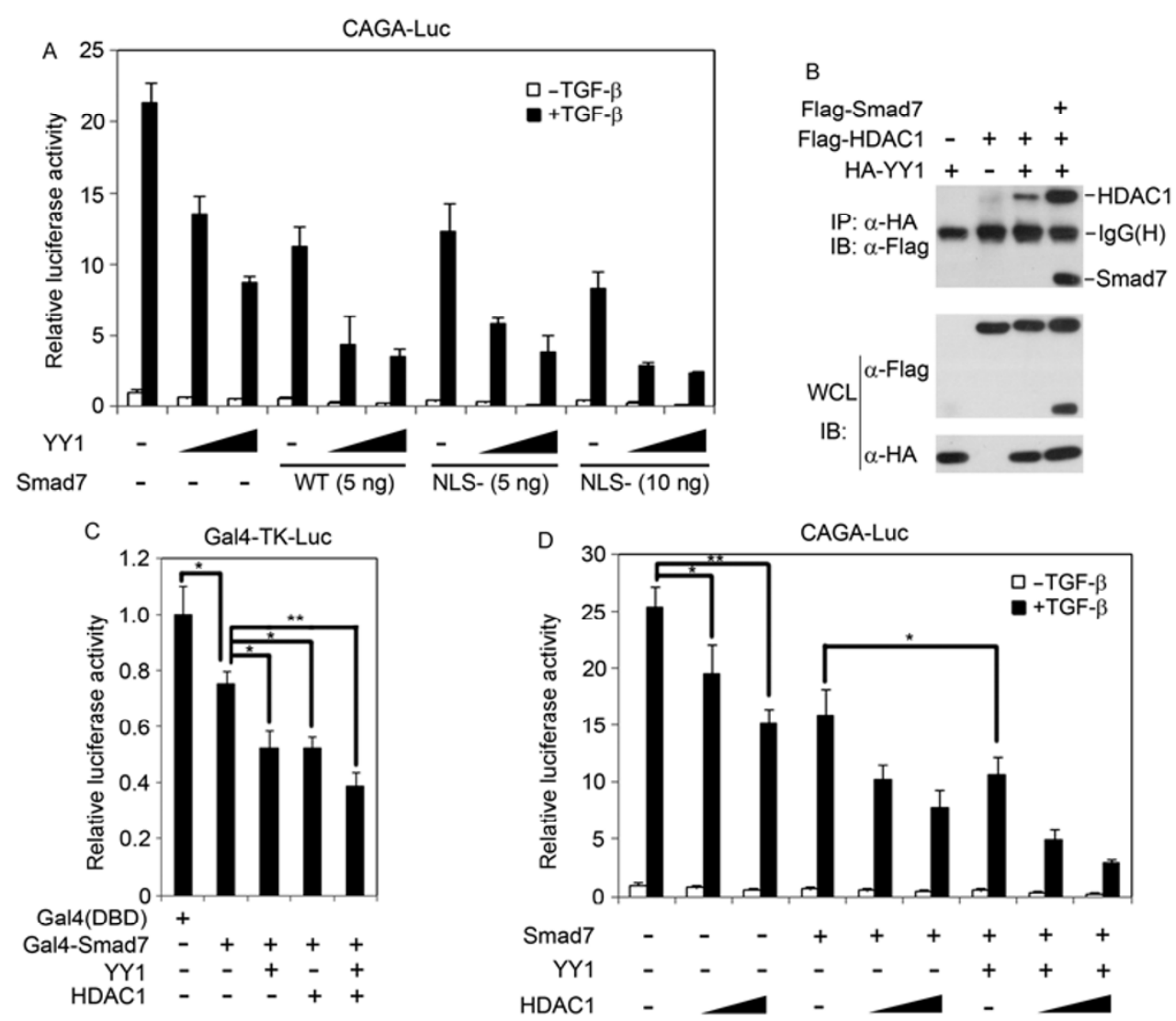

Figure 3 YY1 coordinates with nuclear Smad7 and facilitates its transcription repression activity. A, YY1 coordinates with nuclear Smad7 in antagonizing TGF- $\beta$ signaling. CAGA-luciferase reporter assay was performed as Figure 2A in Hep3B cells. YY1 (10 and $25 \mathrm{ng}$ ) and Smad7 plasmids were transfected as indicated. B, Smad7 enhances YY1 association with HDAC1. IP experiment was done as in Figure 1A in 293FT cells. C, YY1 and HDAC1 facilitate the transcription suppression activity of Smad7. 293FT cells transfected with Gal4-TK-luciferase (200 ng), Renilla (20 ng), Smad7 (100 ng), YY1 (100 ng) or HDAC1 (100 ng) plasmids were subjected to reporter assay and luciferase activity determination. DBD, DNA binding domain of Gal4. *, $P<0.05$; **, $P<0.01$. D, HDAC1 promotes the inhibitory effects of Smad7 and YY1 on TGF- $\beta$ signaling. CAGA-luciferase reporter assay was performed in Hep3B cells. Plasmids encoding Smad7 (5 ng), YY1 (5 ng) and HDAC1 (25 and $50 \mathrm{ng}$ ) were transfected as indicated. *, $P<0.05$; **, $P<0.01$.

and harvested for total RNA extraction and gene expression analysis. As shown in Figure 4B, Smad7 or YY1 alone inhibited expression of several typical TGF- $\beta$ target genes, including PAI-1, FURIN, INHBE, SnoN and FST, and co-expression of Smad7 and YY1 further attenuated their expression.

\section{Discussion}

TGF- $\beta$ /Smad signaling is delicately modulated by numerous regulators in both positive and negative manners [2-5,13]. Two inhibitory Smads, Smad6/7, have been well established as crucial regulators for TGF- $\beta$ superfamily pathways via negative feedback loops [14]. Smad7 is a general antagonist for the TGF- $\beta$ superfamily, while Smad6 is more specifically involved in the BMP pathways. Since its first cloning and characterization in 1997, Smad7 has been extensively studied [46,47]. Gene-targeting and transgenic studies using mouse models have firmly established the crucial roles of Smad7 in embryonic development and adult tissue homeostasis by modulating TGF- $\beta$ signaling [15-18]. Knockout of
Smad7 in mice leads to embryonic lethality or death a few days after birth, depending on gene targeting strategies and mouse background. Smad7-deficient mice showed enhanced phospho-Smad2/3 level in cardiac tissues, B cells or T cells, indicative of augmented TGF- $\beta$ signaling. Moreover, altered expression of Smad7 has been found to be closely related to several human diseases, such as tumorigenesis, scleroderma and chronic inflammatory bowel disease (IBD) [14,15].

Smad7 was first found to form stable complex with TGF- $\beta$ receptors upon TGF- $\beta$ stimulation, inhibiting Smad2/3 recruitment to the receptors and subsequent activation $[46,47]$. Smad7 can recruit WW-HECT type E3 ubiquitin ligases (such as Smurf1, Smurf2, NEDD4-2 or WWP1) to activated T $\beta$ RI, resulting in polyubiquitination and degradation, or the phosphatase GADD34-PP1c to dephosphorylate and inactivate the receptor [14,19]. Furthermore, we have extended Smad7's role by finding that it also impedes TGF- $\beta /$ Smad-induced transcriptional responses in the nucleus [20,21]. Recent studies further emphasized the importance of nuclear Smad7 in regulating gene expression by acting as a co-repressor or co-activator [24-26]. However, the regulatory mechanisms for nuclear Smad7 


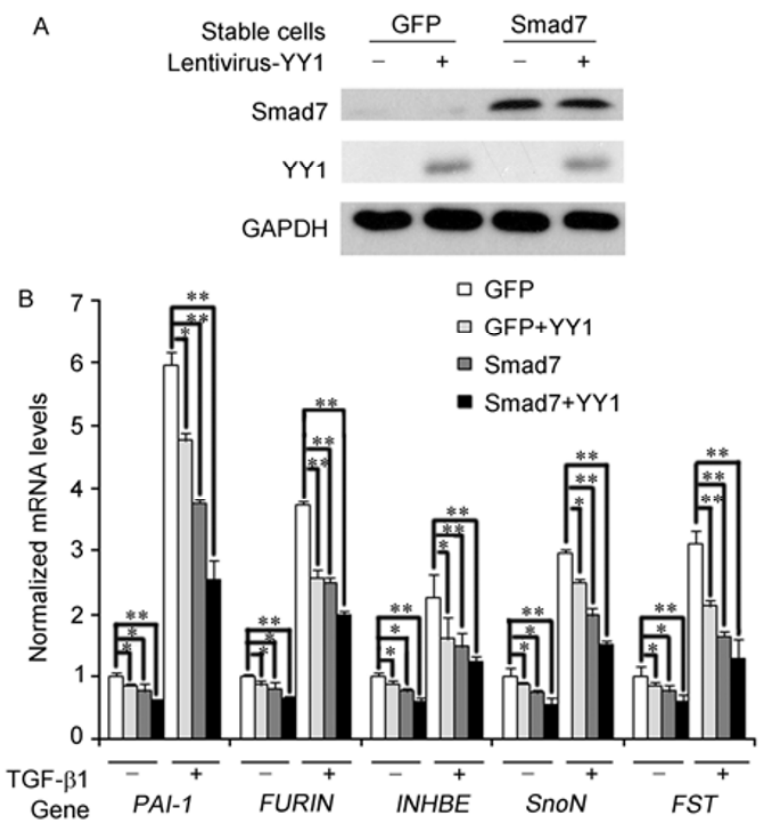

Figure 4 YY1 and Smad7 collaborate with one another to inhibit TGF- $\beta$ target gene expression. A, Expression of YY1 and Smad7 in Hep3B cells. Hep3B cells stably expressing Smad7 were transiently infected with YY1-expression viruses and cultured for $3 \mathrm{~d}$, and analyzed by immunoblotting. B, Parallel cell samples in Figure 4A were treated with or without $100 \mathrm{pmol} \mathrm{L}{ }^{-1}$ TGF- $\beta 1$ for $4 \mathrm{~h}$ before harvested to extract total RNA for gene expression analysis as described in Materials and methods. ${ }^{*}, P<0.05$; **, $P<0.01$.

remain largely unknown. In this study, we found that YY1 was a novel binding partner of Smad7. Overexpression of the two proteins inhibited the TGF- $\beta / S m a d-i n d u c e d$ transcriptional responses in a collaborative manner in both reporter assay and in TGF- $\beta$ target gene expression. In addition, knockdown of endogenous Smad7 alleviated the inhibitory effects of YY1 on TGF- $\beta$ signaling, while silence of YY1 decreased Smad7's effects to a certain degree, implying that the inhibitory function of YY1 on TGF- $\beta$ signaling is dependent on Smad7 to some extent. Interestingly, we noticed that the YY1-Smad7 interaction is attenuated by TGF- $\beta$ treatment, suggesting that their association is signaling-controlled, and could be more significant by acting as a safeguard to prevent basal expression of TGF- $\beta$ targets.

YY1 is a nuclear transcription factor involved in cell growth, differentiation and embryonic development by regulating related gene expression [30-32]. Smad7 is also primarily a nuclear factor, although it may enter the cytoplasm upon TGF- $\beta$ treatment, dependent on cell types or cell lines $[14,20]$. Thereby we proposed that YY1 and Smad7 collaborate in the nucleus. Indeed, functional assays showed that NLS-Smad7 is as effective as wild-type Smad7 in synergizing with YY1 and antagonizing TGF- $\beta$-stimulated reporter expression. As NLS-Smad7 is completely localized in the nucleus when introduced into cells [20], the above results absolutely support their collaboration in the nucleus.
Besides acting as a transcription factor, YY1 can also regulate gene expression without its DNA binding activity, by functioning as a co-factor and recruiting other regulators $[30,32]$. In this regard, the physical interactions between YY1 and R-Smads or Smad4 were found to interfere with Smad-DNA binding, impeding Smad transcriptional activities [37]. Similarly, the CAGA-luciferase and Gal4-TKluciferase reporters used in this study do not have the binding element for YY1 neither, thus YY1 is likely to synergize with Smad7 through physical interactions. Mechanistically, YY1 can interact with HDAC1 and Smad7 simultaneously (Figure 3B), implying that they may form a ternary complex; Smad7 is capable of promoting the YY1-HDAC1 association. Furthermore, YY1 and HDAC1 reinforced the transcription repression ability of Smad7 in Gal4-luciferase reporter assay. HDAC1 is capable of removing acetyl groups from histones, resulting in a more compact conformation of histones and shutdown of gene expression in the vicinity. Therefore, Smad7 and YY1 could act as transcriptional co-repressors to inhibit expression of TGF- $\beta$ target genes. Previous studies showed that Smad7 probably binds DNA elements similar to those of R-Smads or Smad4activin responsive element (ARE) in vitro and the Smad binding element (SBE) in PAI-1 promoter in vivo [20,21]. Although the DNA binding ability of YY1 is not required for inhibiting R-Smads/Smad4-dependent gene expression, or for synergy with $\mathrm{Smad} 7$ in antagonizing the canonical TGF- $\beta$ /Smad signaling, we do not exclude the possibility that the DNA binding abilities of YY1 and Smad7 may play a role in regulating genes other than TGF- $\beta$ targets.

In summary, our results together with previous reports suggest that Smad7 and YY1 could take use of different but related mechanisms to antagonize TGF- $\beta /$ Smad signaling in the nucleus. First, they interfere with functional Smad-DNA complex formation; second, they act as transcriptional co-repressors and inhibit expression of TGF- $\beta$ target genes synergistically by recruiting HDAC1. This newly found cooperative nature of Smad7 and YY1 could play a crucial role in controlling basal expression of TGF- $\beta$ targets. As critical negative regulators, Smad7 could inhibit TGF- $\beta$ / Smad-induced cellular functions such as cell cycle arrest, cell differentiation and apoptosis, and YY1 was also able to block TGF- $\beta /$ Smad-elicited cell differentiation [14,15,37]. Therefore, the functional consequences of Smad7 and YY1 cooperation in antagonizing TGF- $\beta$ / Smad-induced cellular actions remain an open question and deserve further studies.

We are grateful to Drs. Shi Yang (Harvard Medical School), Kee Keh-kooi (Tsinghua University) and Feng XinHua (Baylor College of Medicine) for constructs and other reagents. This work was supported by the National Natural Science Foundation of China (91019003) and National Basic Research Program of China (2011CB943803 and 2013CB933701).

1 Massague J. The transforming growth factor-beta family. Annu Rev Cell Biol, 1990, 6: 597-641 
$2 \mathrm{Xu}$ P, Liu J, Derynck R. Post-translational regulation of TGF-beta receptor and Smad signaling. FEBS Lett, 2012, 586: 1871-1884

3 Moustakas A, Heldin $\mathrm{CH}$. The regulation of TGFbeta signal transduction. Development, 2009, 136: 3699-3714

4 Massague J, Chen YG. Controlling TGF-beta signaling. Genes Dev, 2000, 14: 627-644

5 Massague J. TGFbeta signalling in context. Nat Rev Mol Cell Biol, 2012, 13: 616-630

6 Ikushima H, Miyazono K. Cellular context-dependent "colors" of transforming growth factor-beta signaling. Cancer Sci, 2010, 101: 306-312

7 Heldin $\mathrm{CH}$, Landstrom M, Moustakas A. Mechanism of TGF-beta signaling to growth arrest, apoptosis, and epithelial-mesenchymal transition. Curr Opin Cell Biol, 2009, 21: 166-176

8 Massague J. TGFbeta in cancer. Cell, 2008, 134: 215-230

9 Wu MY, Hill CS. TGF-beta superfamily signaling in embryonic development and homeostasis. Dev Cell, 2009, 16: 329-343

10 Wrighton KH, Feng XH. To (TGF)beta or not to (TGF)beta: fine-tuning of Smad signaling via post-translational modifications. Cell Signal, 2008, 20: 1579-1591

11 Heldin $\mathrm{CH}$, Miyazono K, ten Dijke P. TGF-beta signalling from cell membrane to nucleus through SMAD proteins. Nature, 1997, 390: 465-471

12 Zhang YE. Non-Smad pathways in TGF-beta signaling. Cell Res, 2009, 19: 128-139

13 Feng XH, Derynck R. Specificity and versatility in TGF-beta signaling through Smads. Annu Rev Cell Dev Biol, 2005, 21: 659-693

14 Yan X, Chen YG. Smad7: Not only a regulator, but also a cross-talk mediator of TGF-beta signalling. Biochem J, 2011, 434: 1-10

15 Zhu L, Chen S, Chen Y. Unraveling the biological functions of Smad7 with mouse models. Cell Biosci, 2011, 1: 44

16 Li R, Rosendahl A, Brodin G, Cheng AM, Ahgren A, Sundquist C, Kulkarni S, Pawson T, Heldin CH, Heuchel RL. Deletion of exon I of SMAD7 in mice results in altered B cell responses. J Immunol, 2006, 176: 6777-6784

17 Chen Q, Chen H, Zheng D, Kuang C, Fang H, Zou B, Zhu W, Bu G, Jin T, Wang Z, Zhang X, Chen J, Field LJ, Rubart M, Shou W, Chen Y. Smad7 is required for the development and function of the heart. J Biol Chem, 2009, 284: 292-300

18 Tojo M, Takebe A, Takahashi S, Tanaka K, Imamura T, Miyazono K, Chiba T. Smad7-deficient mice show growth retardation with reduced viability. J Biochem, 2012, 151: 621-631

19 Shi W, Sun C, He B, Xiong W, Shi X, Yao D, Cao X. GADD34-PP1c recruited by Smad7 dephosphorylates TGFbeta type I receptor. J Cell Biol, 2004, 164: 291-300

20 Zhang S, Fei T, Zhang L, Zhang R, Chen F, Ning Y, Han Y, Feng $\mathrm{XH}$, Meng A, Chen YG. Smad7 antagonizes transforming growth factor beta signaling in the nucleus by interfering with functional Smad-DNA complex formation. Mol Cell Biol, 2007, 27: 4488-4499

21 Shi X, Chen F, Yu J, Xu Y, Zhang S, Chen YG, Fang X. Study of interaction between Smad7 and DNA by single-molecule force spectroscopy. Biochem Biophys Res Commun, 2008, 377: 1284-1287

22 Simonsson M, Heldin CH, Ericsson J, Gronroos E. The balance between acetylation and deacetylation controls Smad7 stability. J Biol Chem, 2005, 280: 21797-21803

23 Kume S, Haneda M, Kanasaki K, Sugimoto T, Araki S, Isshiki K, Isono M, Uzu T, Guarente L, Kashiwagi A, Koya D. SIRT1 inhibits transforming growth factor beta-induced apoptosis in glomerular mesangial cells via Smad7 deacetylation. J Biol Chem, 2007, 282: 151-158

24 Emori T, Kitamura K, Okazaki K. Nuclear Smad7 overexpressed in mesenchymal cells acts as a transcriptional corepressor by interacting with HDAC-1 and E2F to regulate cell cycle. Biol Open, 2012, 1: 247-260

25 Miyake T, Alli NS, McDermott JC. Nuclear function of Smad7 promotes myogenesis. Mol Cell Biol, 2010, 30: 722-735
26 Hong S, Kim HY, Kim J, Ha HT, Kim YM, Bae E, Kim TH, Lee KC, Kim SJ. Smad7 protein induces interferon regulatory factor 1-dependent transcriptional activation of caspase 8 to restore tumor necrosis factor-related apoptosis-inducing ligand (TRAIL)-mediated apoptosis. J Biol Chem, 2013, 288: 3560-3570

27 Lin X, Liang YY, Sun B, Liang M, Shi Y, Brunicardi FC, Shi Y, Feng XH. Smad6 recruits transcription corepressor CtBP to repress bone morphogenetic protein-induced transcription. Mol Cell Biol, 2003, 23: 9081-9093

28 Bai S, Shi X, Yang X, Cao X. Smad6 as a transcriptional corepressor. J Biol Chem, 2000, 275: 8267-8270

29 Bai S, Cao X. A nuclear antagonistic mechanism of inhibitory Smads in transforming growth factor-beta signaling. J Biol Chem, 2002, 277: 4176-4182

30 Atchison M, Basu A, Zaprazna K, Papasani M. Mechanisms of Yin Yang 1 in oncogenesis: the importance of indirect effects. Crit Rev Oncog, 2011, 16: 143-161

31 Gordon S, Akopyan G, Garban H, Bonavida B. Transcription factor YY1: structure, function, and therapeutic implications in cancer biology. Oncogene, 2006, 25: 1125-1142

32 Zhang Q, Stovall DB, Inoue K, Sui G. The oncogenic role of Yin Yang 1. Crit Rev Oncog, 2011, 16: 163-197

33 Deng Z, Wan M, Cao P, Rao A, Cramer SD, Sui G. Yin Yang 1 regulates the transcriptional activity of androgen receptor. Oncogene, 2009, 28: 3746-3757

34 Xu J, De Zhu J, Ni M, Wan F, Gu JR. The ATF/CREB site is the key element for transcription of the human RNA methyltransferase like 1(RNMTL1) gene, a newly discovered 17p13.3 gene. Cell Res, 2002, 12: $177-197$

35 Luke MP, Sui G, Liu H, Shi Y. Yin Yang 1 physically interacts with Hoxa11 and represses Hoxa11-dependent transcription. J Biol Chem, 2006, 281: 33226-33232

36 Lu P, Hankel IL, Hostager BS, Swartzendruber JA, Friedman AD, Brenton JL, Rothman PB, Colgan JD. The developmental regulator protein Gon4l associates with protein YY1, co-repressor Sin3a, and histone deacetylase 1 and mediates transcriptional repression. J Biol Chem, 2011, 286: 18311-18319

37 Kurisaki K, Kurisaki A, Valcourt U, Terentiev AA, Pardali K, Ten Dijke P, Heldin CH, Ericsson J, Moustakas A. Nuclear factor YY1 inhibits transforming growth factor beta- and bone morphogenetic protein-induced cell differentiation. Mol Cell Biol, 2003, 23: 4494-4510

38 Lee KH, Evans S, Ruan TY, Lassar AB. SMAD-mediated modulation of YY1 activity regulates the BMP response and cardiac-specific expression of a GATA4/5/6-dependent chick Nkx2.5 enhancer. Development, 2004, 131: 4709v4723

39 Yan X, Lin Z, Chen F, Zhao X, Chen H, Ning Y, Chen YG. Human BAMBI cooperates with Smad7 to inhibit transforming growth factor-beta signaling. J Biol Chem, 2009, 284: 30097-30104

40 Yan X, Zhang J, Sun Q, Tuazon PT, Wu X, Traugh JA, Chen YG. p21-Activated kinase 2 (PAK2) inhibits TGF-beta signaling in Madin-Darby canine kidney (MDCK) epithelial cells by interfering with the receptor-Smad interaction. J Biol Chem, 2012, 287: 13705-13712

41 Yan X, Zhang J, Pan L, Wang P, Xue H, Zhang L, Gao X, Zhao X, Ning Y, Chen YG. TSC-22 promotes transforming growth factor beta-mediated cardiac myofibroblast differentiation by antagonizing Smad7 activity. Mol Cell Biol, 2011, 31: 3700-3709

42 Kee K, Angeles VT, Flores M, Nguyen HN, Reijo Pera RA. Human DAZL, DAZ and BOULE genes modulate primordial germ-cell and haploid gamete formation. Nature, 2009, 462: 222-225

43 Chen YG, Liu F, Massague J. Mechanism of TGFbeta receptor inhibition by FKBP12. Embo J, 1997, 16: 3866-3876

44 Dennler S, Itoh S, Vivien D, ten Dijke P, Huet S, Gauthier JM. Direct binding of Smad3 and Smad4 to critical TGF beta-inducible elements in the promoter of human plasminogen activator inhibitor-type 1 gene. EMBO J, 1998, 17: 3091-3100 
45 Pulaski L, Landstrom M, Heldin CH, Souchelnytskyi S. Phosphorylation of Smad7 at Ser-249 does not interfere with its inhibitory role in transforming growth factor-beta-dependent signaling but affects Smad7-dependent transcriptional activation. J Biol Chem, 2001, 276: 14344-14349

46 Nakao A, Afrakhte M, Moren A, Nakayama T, Christian JL, Heuchel R, Itoh S, Kawabata M, Heldin NE, Heldin CH, ten Dijke P. Identifi- cation of Smad7, a TGFbeta-inducible antagonist of TGF-beta signalling. Nature, 1997, 389: 631-635

47 Hayashi H, Abdollah S, Qiu Y, Cai J, Xu YY, Grinnell BW, Richardson MA, Topper JN, Gimbrone MA Jr., Wrana JL, Falb D. The MAD-related protein Smad7 associates with the TGF $\beta$ receptor and functions as an antagonist of TGF $\beta$ signaling. Cell, 1997, 89: 1165-1173

Open Access This article is distributed under the terms of the Creative Commons Attribution License which permits any use, distribution, and reproduction in any medium, provided the original author(s) and source are credited. 